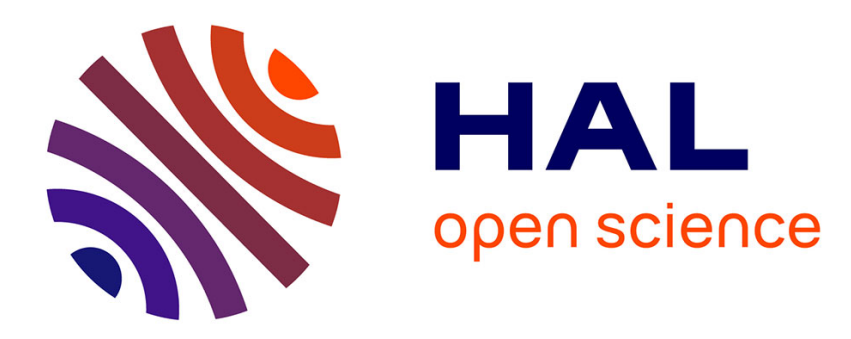

\title{
Upper Bounds on Betti Numbers of Tropical Prevarieties
}

\author{
Dima Grigoriev, Nicolai Vorobjov
}

\section{To cite this version:}

Dima Grigoriev, Nicolai Vorobjov. Upper Bounds on Betti Numbers of Tropical Prevarieties. Arnold Mathematical Journal, 2018, 4, pp.127 - 136. 10.1007/s40598-018-0086-1 . hal-03043493

\section{HAL Id: hal-03043493 https://hal.science/hal-03043493}

Submitted on 7 Dec 2020

HAL is a multi-disciplinary open access archive for the deposit and dissemination of scientific research documents, whether they are published or not. The documents may come from teaching and research institutions in France or abroad, or from public or private research centers.
L'archive ouverte pluridisciplinaire HAL, est destinée au dépôt et à la diffusion de documents scientifiques de niveau recherche, publiés ou non, émanant des établissements d'enseignement et de recherche français ou étrangers, des laboratoires publics ou privés. 


\title{
Upper Bounds on Betti Numbers of Tropical Prevarieties
}

\author{
Dima Grigoriev $^{1}$ • Nicolai Vorobjov ${ }^{2}$
}

Received: 5 October 2017 / Revised: 9 March 2018 / Accepted: 12 March 2018

(C) Institute for Mathematical Sciences (IMS), Stony Brook University, NY 2018

\begin{abstract}
We prove upper bounds on the sum of Betti numbers of tropical prevarieties in dense and sparse settings. In the dense setting the bound is in terms of the volume of Minkowski sum of Newton polytopes of defining tropical polynomials, or, alternatively, via the maximal degree of these polynomials. In sparse setting, the bound involves the number of the monomials.
\end{abstract}

Keywords Tropical prevariety $\cdot$ Betti numbers $\cdot$ Polyhedral complex

Mathematics Subject Classification 14T05

\section{Introduction}

In this paper we are concerned with upper bounds on Betti numbers of tropical prevarieties. Basic definitions and statements regarding tropical algebra and geometry can be found in Maclagan and Sturmfels (2015) and Richter-Gebert et al. (2005).

Each tropical polynomial $f$ in $n$ variables can be represented as $\min \left\{L_{1}, \ldots, L_{m}\right\}$, where $L_{1}, \ldots, L_{m}$ are linear functions on $\mathbb{R}^{n}$ (called tropical monomials) with nonnegative integer coefficients at variables and real constant terms (if negative integer coefficients are allowed, then $f$ is called tropical Laurent polynomial). For a monomial $L_{j}$ its degree is the sum of integer coefficients at variables, the maximum among

\section{Nicolai Vorobjov \\ nnv@cs.bath.ac.uk \\ Dima Grigoriev \\ dmitry.grigoryev@math.univ-lille1.fr}

1 CNRS, Mathématiques, Université de Lille, 59655 Villeneuve d'Ascq, France

2 Department of Computer Science, University of Bath, Bath, England BA2 7AY, UK 
degrees of the monomials is called the (tropical) degree of $f$. With any tropical polynomial $f$ we associate a concave piece-wise linear function

$$
L(\mathbf{x}):=\min _{1 \leq j \leq m}\left\{L_{j}(\mathbf{x})\right\}
$$

defined on $\mathbb{R}^{n}$. A tropical hypersurface $V:=V(f) \subset \mathbb{R}^{n}$ is the set of all points in $\mathbb{R}^{n}$ at which $L(\mathbf{x})$ is not smooth. Any point $\mathbf{x} \in V$ is called a zero of $f$. A tropical prevariety is an intersection of a finite number of tropical hypersurfaces, in other words, the set of all common tropical zeroes of a finite system of multivariate tropical polynomials.

Let $V:=V\left(f_{1}, \ldots, f_{k}\right) \subset \mathbb{R}^{n}$ be the tropical prevariety defined by a system $f_{1}, \ldots, f_{k}$ of tropical polynomials in $n$ variables of degrees not exceeding $d$. In case $k=n$, the Tropical Bezout Theorem (Richter-Gebert et al. 2005) states that the number of all stable tropical zeroes (counted with multiplicities) of the system $f_{1}, \ldots, f_{k}$ is the product of the degrees of its polynomials. For arbitrary $k$, the upper bound

$$
\left(\begin{array}{c}
k+7 n \\
3 n
\end{array}\right) d^{3 n}
$$

on the number of connected components of $V$ was obtained in Davydow and Grigoriev (2017). In case $k \leq n$, the number of maximal faces of transversal intersections of tropical hypersurfaces defined by polynomials $f_{i}, 1 \leq i \leq k$, was expressed in Bertrand and Bihan (2013), Steffens and Theobald (2010) in terms of mixed Minkowski volumes of Newton polytopes of polynomials $f_{i}$. In Bihan (2016), for $k=n$, this number was bounded from above using a new concept of discrete mixed volume for sparse tropical polynomials.

The structure of this paper is as follows.

In Sect. 2 we show that any homotopy type of a polyhedral complex is realizable by a tropical prevariety, so the problem of bounds on Betti numbers is not futile.

In Sect. 3 we prove the upper bound

$$
2^{(r+1)} r ! \operatorname{Vol}_{r}\left(P_{1}+\cdots+P_{k}\right)
$$

on the sum of Betti numbers of a tropical prevariety $V$, where $r$ is the dimension of the Minkowski sum $P_{1}+\cdots+P_{k}$ of Newton polytopes $P_{1}, \ldots, P_{k}$ of tropical polynomials $f_{1}, \ldots, f_{k}$, and $\operatorname{Vol}_{r}\left(P_{1}+\cdots+P_{k}\right)$ is the $r$-dimensional volume of the Minkowski sum. In terms of $\max _{i} \operatorname{deg} f_{i}=d$ this implies the bound

$$
2^{(n+1)}(k d)^{n} \text {. }
$$

Note that in Davydow and Grigoriev (2017) a naive upper bound

$$
\left(\left(\begin{array}{c}
k+7 n \\
3 n
\end{array}\right) d^{3 n}\right)^{n}
$$

was mentioned. Also in Davydow and Grigoriev (2017), an example is constructed of a prevariety defined by $k n$ polynomials of degrees at most $d$, containing $(k d / 4)^{n}$ 
zero-dimensional connected components. Comparing this lower bound with (1.1), we note a gap within a factor $n^{n}$. An interesting challenge is to close this gap.

In Sect. 4 we assume that each tropical polynomial $f_{i}, 1 \leq i \leq k$ is $m$-sparse, i.e., consists of at most $m$ monomials. In this setting we prove the upper bound

$$
n 2^{n}\left(\begin{array}{c}
k\left(\begin{array}{c}
m \\
2
\end{array}\right) \\
n
\end{array}\right)
$$

on the sum of Betti numbers of $V$. We give an example of a prevariety, with $k=n$, for which this bound is close to sharp up to the factor $m^{n}$.

Results in Sect. 3 can be related to the classical upper bounds on Betti numbers of real algebraic and semi-algebraic sets obtained by Petrovskii, Oleinik, Milnor and Thom, and developed further by various authors. In particular, Milnor (1964) proved that if a semi-algebraic set $X \subset \mathbb{R}^{n}$ is defined by a system of $k$ non-strict polynomial inequalities of degrees less than $d$, then the sum of Betti numbers of $X$ is at most $(c k d)^{n}$ for an absolute constant $c>0$ (compare with (1.1)). Note that in the case when $X$ is defined by an arbitrary Boolean combination of inequalities, the $i$ th Betti number of $X$ does not exceed $(c v k d)^{n}$ for $v=\min \{i+1, n-i, k\}$ and an absolute constant $c>0$ (Gabrielov and Vorobjov 2009).

The bound in Sect. 4 can be viewed as a tropical counterpart of the bounds on Betti numbers for fewnomials (Khovanskii 1991; Gabrielov and Vorobjov 2004; Bihan and Sottile 2009).

In Appendix we prove a technical statement needed to reduce the polyhedral complex associated with a prevariety $V$ to a bounded polyhedral complex.

\section{Tropical Prevarieties and Polyhedral Complexes}

It is well known (Maclagan and Sturmfels 2015; Richter-Gebert et al. 2005) that any tropical prevariety $V:=V\left(f_{1}, \ldots, f_{k}\right) \subset \mathbb{R}^{n}$ of all common tropical zeroes of tropical polynomials $f_{1}, \ldots, f_{k}$ is the union of cells of a finite polyhedral complex in $\mathbb{R}^{n}$ (in Lemmas 4.1, 4.3 below, we provide an explicit representation of $V$ as a complex). For this complex we will use the same notation, $V$. There is an understanding among experts (see, e.g., Bogart et al. 2007) that any homotopy type of a polyhedral complex is realizable by a tropical prevariety. In the present section we make this statement precise.

Let $A \varsubsetneqq \mathbb{R}^{n}$ be an affine subspace, defined by linear equations with integer coefficients, and $A_{\geq}$be a closed half-space in $A$ defined by a non-strict linear inequality with integer coefficients.

Lemma 2.1 The half-space $A_{\geq}$is a tropical prevariety in $\mathbb{R}^{n}$.

Proof Let $A \varsubsetneqq \mathbb{R}^{n}$ be defined by a system of equations $M_{i}=0,1 \leq i \leq s$, and $A_{\geq}$ by a system $M_{i}=0,1 \leq i \leq s, L \geq 0$, where all $M_{i}, L$ are linear polynomials with integer coefficients. Then $A_{\geq}=V\left(f_{1}, \ldots, f_{s}, f_{s+1}\right)$, where $f_{i}, 1 \leq i \leq s+1$, are tropical Laurent polynomials such that $f_{i}, 1 \leq i \leq s$, is represented as $\min \left\{M_{i}, 0\right\}$, 
while $f_{s+1}$ as $\min \left\{0, M_{1}, L\right\}$. By adding an appropriate linear polynomial to monomials in $f_{i}$, we turn $f_{i}$ into a tropical polynomial having the same zeroes as $f_{i}$.

Corollary 2.2 Any finite union of rational convex polyhedra (in particular, the union of all cells in any rational polyhedral complex) of positive codimension in $\mathbb{R}^{n}$ coincides with a tropical prevariety in $\mathbb{R}^{n}$.

Proof By Lemma 2.1, any rational convex polyhedron of positive codimension in $\mathbb{R}^{n}$ is a tropical prevariety. It remains to note that the union of a finite number of tropical prevarieties is also a tropical prevariety (this is proved by a straightforward analogy with the classical case).

As another consequence of Lemma 2.1 we mention the following example of a countable family of tropical prevarieties such that their intersection is not a tropical prevariety. A standard 2-dimensional disk in a 2-dimensional subspace of $\mathbb{R}^{3}$ is not a tropical prevariety, but can be approximated from outside by a shrinking countable family of rational convex polyhedra.

\section{Betti Numbers for Dense Tropical Polynomials}

Let a tropical polynomial $f$ be represented as

$$
\min _{1 \leq j \leq m}\left\{\sum_{1 \leq i \leq n} a_{j i} x_{i}+b_{j}\right\},
$$

where $0 \leq a_{j i} \in \mathbb{Z}$ and $b_{j} \in \mathbb{R}$. The Newton polytope $P \subset \mathbb{R}^{n}$ of $f$ is the convex hull of $m$ points $\left(a_{j 1}, \ldots, a_{j n}\right), 1 \leq j \leq m$ (see, e.g., Maclagan and Sturmfels 2015).

Let $E \subset \mathbb{R}^{n+1}$ be the convex hull of $m$ points $\left(a_{j 1}, \ldots, a_{j n}, b_{j}\right), 1 \leq j \leq m$. The extended Newton polytope of $f$ is the set

$$
Q:=E+\{(0, \ldots, 0, c) \mid 0 \leq c \in \mathbb{R}\} \subset \mathbb{R}^{n+1},
$$

Consider a tropical prevariety $V:=V\left(f_{1}, \ldots, f_{k}\right) \subset \mathbb{R}^{n}$. Let $P_{i} \subset \mathbb{R}^{n}, 1 \leq i \leq k$, be the Newton polytope of $f_{i}, r$ be the dimension of the Minkowski sum $P_{1}+\cdots+P_{k}$, and $\operatorname{Vol}_{r}\left(P_{1}+\cdots+P_{k}\right)$ be its $r$-dimensional volume. Without loss of generality, we assume that this volume is positive.

Theorem 3.1 The number of faces of all dimensions of $V$ does not exceed

$$
\left(2^{r+1}-1\right) r ! \operatorname{Vol}_{r}\left(P_{1}+\cdots+P_{k}\right) .
$$

Before proving this theorem, let us extract some corollaries regarding bounds on Betti numbers.

According to Theorem 5.1 in Appendix, the prevariety $V$, being a finite polyhedral complex, is homotopy equivalent to a bounded polyhedral complex $W$, having no more 
cells than $V$. Note that $W$ has the structure of a finite $\mathrm{CW}$ complex with polyhedral cells. Let $\varphi(W)$ be the number of all faces of $W$. Then, by Theorem 3.1,

$$
\varphi(W) \leq\left(2^{r+1}-1\right) r ! \operatorname{Vol}_{r}\left(P_{1}+\cdots+P_{k}\right) .
$$

We will use notations $b_{v}(X):=\operatorname{rank} H_{v}(X, \mathbb{R})$, where $H_{\nu}(X, \mathbb{R})$ is a singular $v$ th homology group, and

$$
\mathrm{b}(X):=\sum_{0 \leq \nu \leq \operatorname{dim} X} \mathrm{~b}_{\nu}(X)
$$

Recalling that $\mathrm{b}(V)=\mathrm{b}(W)$, and applying to the CW complex $W$ the Weak Morse Inequality $\mathrm{b}(W) \leq \varphi(W)$ (Forman 1998, Corollary 3.7) we get from (3.1) the following upper bound.

Corollary 3.2 The sum of Betti numbers of $V$ satisfies the inequality

$$
\mathrm{b}(V) \leq\left(2^{r+1}-1\right) r ! \operatorname{Vol}_{r}\left(P_{1}+\cdots+P_{k}\right) .
$$

Let $d=\max _{1 \leq i \leq k} \operatorname{deg} f_{i}$. Then each $P_{i}$ is contained in the simplex

$$
\left\{\left(x_{1}, \ldots, x_{n}\right) \in \mathbb{R}^{n} \mid x_{j} \geq 0,1 \leq j \leq n, x_{1}+\cdots+x_{n} \leq d\right\} .
$$

Hence, in terms of $d$, the inequality (3.2) can be presented in the following form.

Corollary 3.3 The sum of Betti numbers of $V$ satisfies the inequality

$$
\mathrm{b}(V) \leq\left(2^{n+1}-1\right)(k d)^{n}
$$

Proof of Theorem 3.1 Let $Q_{i} \subset \mathbb{R}^{n+1}, 1 \leq i \leq k$ be the extended Newton polytope of $f_{i}$. Let $Q$ be the bottom of $Q_{1}+\cdots+Q_{k}$, which is the set of all points $(\mathbf{x}, a) \in$ $Q_{1}+\cdots+Q_{k}$ such that there are no points $(\mathbf{x}, b) \in Q_{1}+\cdots+Q_{k}$ with $b<a$. Note that for the projection map $\pi: \mathbb{R}^{n+1} \rightarrow \mathbb{R}^{n}$ along the last coordinate we have $\pi(Q)=\pi\left(Q_{1}+\cdots+Q_{k}\right)=P_{1}+\cdots+P_{k}$. The restriction $\left.\pi\right|_{Q}$ is injective, $\operatorname{dim}(Q)=\operatorname{dim}\left(P_{1}+\cdots+P_{k}\right)=r$.

Let $F$ be a face of $Q$. Its dual, $G(F)$, is the set of all supporting hyperplanes for $Q$ (subset of the set of all supporting hyperplanes for $Q_{1}+\cdots+Q_{k}$ ) such that their intersections with $Q$ coincide with $F$, and no hyperplane contains a straight line parallel to $\{(0, \ldots, 0, c\} \mid c \in \mathbb{R}\}$. Then $G(F)$ can be identified with a face of the dual polytope to $Q_{1}+\cdots+Q_{k}$, and we have $\operatorname{dim} F+\operatorname{dim} G(F)=n$ (see, e.g., Bertrand and Bihan 2013; Bihan 2016; Steffens and Theobald 2010). Observe that $F$ is representable as a Minkowski sum $F=F_{1}+\cdots+F_{k}$, where each $F_{i}$ is a face of the bottom of $Q_{i}$, and $H \cap Q_{i}=F_{i}$ for any $H \in G(F)$. We say that a face $F$ of $Q$ is tropical if $\operatorname{dim} F_{i} \geq 1$ for all $1 \leq i \leq k$. Then $V$ coincides with the union of polytopes $G(F)$ for all tropical faces $F$ (cf. Bertrand and Bihan 2013; Steffens and Theobald 2010). 
Decompose each $r$-dimensional face of $Q$ into $r$-dimensional closed simplices without adding new vertices, and obtain a triangulation of $Q$ (cf. Prasolov 2006, Theorem 8.2). The number of all simplices in this triangulation is not less than the total number of faces of $V$. For each $r$-dimensional simplex $S$ in the triangulation the $r$-dimensional simplex $\pi(S)$ has integer vertices. Since the volume of any $r$ dimensional simplex with integer vertices in $\mathbb{R}^{n}$ is at least $1 / r$ !, we conclude that $\operatorname{Vol}_{r}(\pi(S)) \geq 1 / r$ !. Therefore, the number of all $r$-dimensional simplices in the triangulation does not exceed $r$ ! $\operatorname{Vol}_{r}\left(P_{1}+\cdots+P_{k}\right)$. To complete the proof, it remains to notice that the number of all faces of an $r$-dimensional simplex is $2^{r+1}-1$.

\section{Betti Numbers for Sparse Tropical Polynomials}

In this section we assume that each tropical polynomial $f_{i}, 1 \leq i \leq k$ is $m$-sparse, i.e., contains at most $m$ monomials. In other words, each $f_{i}$ can be represented as $\min \left\{L_{i, 1}, \ldots, L_{i, m}\right\}$, where $L_{i, 1}, \ldots, L_{i, m}$ are linear functions on $\mathbb{R}^{n}$. Although, by definition, coefficients in $L_{i, j}$ at variables are non-negative integers, all results in this section hold for arbitrary real coefficients.

Following Grigoriev and Podolskii (2015), for any subset $B$ of $D:=\{(i, j) \mid 1 \leq$ $i \leq k, 1 \leq j \leq m\}$, consider the polyhedron $U_{B}$ consisting of all points $\mathbf{x} \in \mathbb{R}^{n}$ such that

$$
\min _{1 \leq j \leq m}\left\{L_{i, j}(\mathbf{x})\right\}=L_{i, j_{0}}(\mathbf{x}) \quad \text { for every }\left(i, j_{0}\right) \in B
$$

and

$$
\min _{1 \leq j \leq m}\left\{L_{i, j}(\mathbf{x})\right\}<L_{i, j_{1}}(\mathbf{x}) \text { for every }\left(i, j_{1}\right) \notin B
$$

Note that each set $U_{B}$ is open in its linear hull, and $U_{B} \subset V$ if and only if for each $1 \leq i \leq k$ there exist $1 \leq j_{0}<j_{1} \leq m$ such that $\left(i, j_{0}\right),\left(i, j_{1}\right) \in B$.

Denote by $\mathcal{L}(X)$ the linear hull of a set $X \subset \mathbb{R}^{n}$.

Lemma 4.1 The interior of every face $F$ of $U_{B}$ in $\mathcal{L}(F)$ coincides with $U_{B_{1}}$ for a suitable subset $B_{1} \subset D$ such that $B \varsubsetneqq B_{1}$.

Proof There exists a subset $B_{1} \subset D$ such that $\operatorname{dim}\left(U_{B_{1}} \cap F\right)=\operatorname{dim}(F)$. We prove that $B_{1}$ satisfies the requirements of the lemma. Observe that $U_{B_{1}}$ is contained in $\mathcal{L}(F)$. The condition $\min _{j}\left\{L_{i, j}(\mathbf{x})\right\}=L_{i, j_{0}}(\mathbf{x})$ for every $\left(i, j_{0}\right) \in B$ and each $\mathbf{x} \in \mathcal{L}\left(U_{B}\right)$ implies that $\left(i, j_{0}\right) \in B_{1}$. Hence, $B \subset B_{1}$, and obviously $B \neq B_{1}$.

By Davydow and Grigoriev (2017, Theorem 4.4), for any two points in $U_{B_{1}}$, their sufficiently small neighbourhoods are homeomorphic (in fact, isomorphic) by a linear translation from one point to another. Therefore, $U_{B_{1}}$ is contained in the interior of $F$ in $\mathcal{L}(F)$. It remains to show that, conversely, $U_{B_{1}}$ contains the interior of $F$.

For contradiction, assume that there exists a point $\mathbf{x}$ in the interior of $F$ such that $\mathbf{x} \in$ $\overline{U_{B_{1}}} \cap U_{B_{2}}$ for some subset $B_{2} \subset D$ different from $B_{1}$, where $\overline{U_{B_{1}}}$ denotes the closure of $U_{B_{1}}$. Choose any $\left(i, j_{2}\right) \in B_{2} \backslash B_{1}$, and $\left(i, j_{0}\right) \in B$ such that $\min _{j}\left\{L_{i, j}(\mathbf{y})\right\}=L_{i, j_{0}}(\mathbf{y})$ 
for every $\mathbf{y} \in \mathcal{L}\left(U_{B}\right)$. It follows that $\min _{j}\left\{L_{i, j}(\mathbf{y})\right\}=L_{i, j_{2}}(\mathbf{y})$ for every $\mathbf{y} \in \mathcal{L}(F)$. We get a contradiction with the assumption that $\left(i, j_{2}\right) \notin B_{1}$, hence $U_{B_{1}}$ contains the interior of $F$ in $\mathcal{L}(F)$.

Remark 4.2 Being a polyhedron, the set $\overline{U_{B}}$ coincides with

$$
\left\{\mathbf{x} \in \mathbb{R}^{n} \mid \min _{j}\left\{L_{i, j}(\mathbf{x})\right\}=L_{i, j_{0}}(\mathbf{x}), 1 \leq i \leq k \text { for every }\left(i, j_{0}\right) \in B\right\} .
$$

Lemma 4.3 For any subsets $B_{1}, B_{2} \subset D$ there exists a subset $B \subset D$ such that $B \supset\left(B_{1} \cup B_{2}\right)$ and $\overline{U_{B}}=\overline{U_{B_{1}}} \cap \overline{U_{B_{2}}}$.

Proof For any $\left(i, j_{0}\right) \in B_{1} \cup B_{2}, 1 \leq i \leq k$ and any $\mathbf{x} \in \overline{U_{B_{1}}} \cap \overline{U_{B_{2}}}$ we have $\min _{j}\left\{L_{i, j}(\mathbf{x})\right\}=L_{i, j_{0}}(\mathbf{x})$.

Define $B$ as the set of all $\left(i, j_{1}\right) \in D$ such that $\min _{j}\left\{L_{i, j}(\mathbf{x})\right\}=L_{i, j_{1}}(\mathbf{x})$ for every $\mathbf{x} \in \overline{U_{B_{1}}} \cap \overline{U_{B_{2}}}$. Hence, $B \supset\left(B_{1} \cup B_{2}\right)$. It remains to prove that $\overline{U_{B}}=\overline{U_{B_{1}}} \cap \overline{U_{B_{2}}}$.

The inclusion $\overline{U_{B}} \subset\left(\overline{U_{B_{1}}} \cap \overline{U_{B_{2}}}\right)$ follows from Remark 4.2. Conversely, since $\overline{U_{B_{1}}} \cap \overline{U_{B_{2}}}$ is a closed convex polyhedron, for every $\left(i, j_{2}\right) \in D \backslash B$ the set of all $\mathbf{x} \in \overline{U_{B_{1}}} \cap \overline{U_{B_{2}}}$ such that $\min _{j}\left\{L_{i, j}(\mathbf{x})\right\}<L_{i, j_{2}}(\mathbf{x})$ contains the interior of $\overline{U_{B_{1}}} \cap \overline{U_{B_{2}}}$. Hence, $U_{B}$ also contains this interior. It follows that $\left(\overline{U_{B_{1}}} \cap \overline{U_{B_{2}}}\right) \subset \overline{U_{B}}$.

Similar to Davydow and Grigoriev (2017), consider an arrangement $\mathcal{A}$ in $\mathbb{R}^{n}$ consisting of at most $\ell:=k\left(\begin{array}{c}m \\ 2\end{array}\right)$ hyperplanes of the form $L_{i, j_{1}}=L_{i, j_{2}}$ for all $1 \leq i \leq k, 1 \leq j_{1}<j_{2} \leq m$. Without loss of generality, assume that $n \leq \ell$.

Observe that for every $B \subset D$ the set $U_{B}$ is a face of $\mathcal{A}$ whenever $U_{B} \neq \varnothing$. Then Lemmas 4.1 and 4.3 imply that the number of faces $\varphi(V)$ of $V$ does not exceed the number of faces $\varphi(\mathcal{A})$ of $\mathcal{A}$. According to Zaslavski (1975), $\varphi(\mathcal{A}) \leq n 2^{n}\left(\begin{array}{l}\ell \\ n\end{array}\right)$, thus $\varphi(V) \leq n 2^{n}\left(\begin{array}{l}\ell \\ n\end{array}\right)$. We proved the following theorem.

Theorem 4.4 The number of all faces of a tropical prevariety $V \subset \mathbb{R}^{n}$ defined by $k$ $m$-sparse tropical polynomials is at most

$$
n 2^{n}\left(\begin{array}{c}
k\left(\begin{array}{c}
m \\
2
\end{array}\right) \\
n
\end{array}\right)
$$

As in the proof of Corollary 3.2, passing from $V$ to a homotopy equivalent bounded polyhedral complex $W$, having no more cells than $V$ has, we obtain the following corollary.

Corollary 4.5 The sum of Betti numbers of $V$ satisfies the inequality

$$
\mathrm{b}(V) \leq n 2^{n}\left(\begin{array}{c}
k\left(\begin{array}{c}
m \\
2
\end{array}\right) \\
n
\end{array}\right)
$$

In conclusion, we construct an example of a tropical prevariety with $k=n$ which shows that upper bounds in Theorem 4.4 and Corollary 4.5 differ from a lower bound up to a factor $m^{n}$. 
Let $f_{i}, 1 \leq i \leq n$ be a tropical polynomial in one variable $X_{i}$, of degree $m$ with $m$ tropical zeroes. Then the tropical prevariety defined by the system $f_{1}, \ldots, f_{n}$ consists of exactly $m^{n}$ isolated points.

\section{Appendix: Homotopy Equivalence of a Finite Polyhedral Complex to a Bounded Polyhedral Complex}

Theorem 5.1 Let $K$ be a finite polyhedral complex in $\mathbb{R}^{n}$ in which some cells may be unbounded. Then $K$ is homotopy equivalent to a bounded polyhedral complex having no more cells than $K$ has.

Lemma 5.2 If a convex (closed) polyhedron $P$ in $\mathbb{R}^{n}$ contains a straight line $\ell$, and $T$ is an affine subspace $T$ in $\mathbb{R}^{n}$, complementary orthogonal to $\ell$, then $P \simeq \ell \times(P \cap T)$, where $\simeq$ denotes homeomorphism.

Proof Observe that for any $\mathbf{x} \in P$, in particular, for any $\mathbf{x} \in P \cap T$, the straight line $m$ through $\mathbf{x}$, parallel to $\ell$, is also contained in $P$.

Lemma 5.3 Let $K$ be a finite polyhedral complex in $\mathbb{R}^{n}$, with a cell containing a straight line $\ell$, and $T$ be an affine subspace $T$ in $\mathbb{R}^{n}$, complementary orthogonal to $\ell$. Then the connected component $C$ of $K$, containing $\ell$ is homeomorphic to $\ell \times(C \cap T)$, where $C \cap T$ is a $(\operatorname{dim}(K)-1)$-dimensional connected polyhedral complex consisting of all cells of the kind $P \cap T$ for all cells $P$ in $C$.

Proof Let $P$ and $Q$ be the cells in $K$, having a common face $R$, and let $P$ contain a straight line $\ell$. By Lemma 5.2, $P \simeq \ell \times(P \cap T)$. Since $R \subset Q$, cells $Q$ and $R$ contain straight lines $\ell_{Q}$ and $\ell_{R}$, respectively, parallel to $\ell$. Hence $Q \simeq \ell \times(Q \cap T)$ and $R \simeq \ell \times(R \cap T)$. It follows that $P$ and $Q$ are in the same connected component $C$ of $K$ and $P \cup Q \simeq \ell \times\left(P^{\prime} \cup Q^{\prime}\right)$.

Lemma 5.4 Let $K$ be a finite polyhedral complex in $\mathbb{R}^{n}$, with a cell D containing an affine space $L$ with $\operatorname{dim}(L)=d \geq 0$ and not containing any affine space of dimension greater than $d$. Let $T$ be an affine space complementary orthogonal to $L$. Then the connected component $C$ of $K$, containing $D$, is homeomorphic to $L \times(C \cap T)$, where $C \cap T$ is a $(\operatorname{dim}(K)-d)$-dimensional connected polyhedral complex, not containing any straight line, and consisting of all cells of the kind $P \cap T$ for all cells $P$ in $C$.

Proof Proof by induction on $d$, with the base for $d=0$ being obvious. If $d>0$ then $D$ contains a straight line, which we denote by $\ell$. Let $T_{\ell}$ be an affine space complementary orthogonal to $\ell$. By Lemma 5.3, $C \simeq \ell \times\left(C \cap T_{\ell}\right)$, while $\operatorname{dim}\left(C \cap T_{\ell}\right)=\operatorname{dim}(C)-1$. The cell $D^{\prime}:=D \cap T_{\ell}$ contains the affine space $L \cap T_{\ell}, \operatorname{dim}\left(L \cap T_{\ell}\right)=d-1$, and does not contain any affine space of dimension greater than $d-1$. To conclude the proof, apply the inductive hypothesis to $C \cap T_{\ell}$.

Lemma 5.5 Any finite polyhedral complex $K$ in $\mathbb{R}^{n}$, is homotopy equivalent to a complex $K^{\prime}$ in $\mathbb{R}^{n}$ in which no cell contains a straight line. Complexes $K$ and $K^{\prime}$ have equal number of cells. 
Proof Consider in turn each connected component $C$ of $K$. Let $C$ contain an affine space $L_{C}$ with $\operatorname{dim}\left(L_{C}\right) \geq 0$ and not contain any affine space of dimension greater than $\operatorname{dim}\left(L_{C}\right)$. By Lemma 5.4, $C \simeq L_{C} \times\left(C \cap T_{C}\right)$, where $T_{C}$ is an affine space complementary orthogonal to $L_{C}$. Then $C^{\prime}:=C \cap T_{C}$ is a deformation retract of $C$, not containing a straight line. Moreover, each cell in $C^{\prime}$ is of the form $D \cap T_{C}$, where $D$ is a cell in $C$, hence complexes $C$ and $C^{\prime}$ have equal number of cells.

Proof of Theorem 5.1 In view of Lemma 5.5, it is sufficient to prove the theorem in the case when no cell in $K$ contains a straight line. In particular, every connected component of $K$ is pointed (i.e., contains a vertex of $K$ ).

Semi-algebraic local triviality (Hardt 1980) implies that for any sufficiently large positive $\rho \in \mathbb{R}$ the intersection $\widehat{K}:=K \cap S_{\rho}$, and the intersection of $K$ with the interior of $S_{\rho}$, for the simplex

$$
S_{\rho}=\left\{\left(x_{1}, \ldots, x_{n}\right) \in \mathbb{R}^{n} \mid x_{j} \geq-\rho, 1 \leq j \leq n, x_{1}+\cdots+x_{n} \leq \rho\right\}
$$

are both homotopy equivalent to $K$. Impose on $\widehat{K}$ a structure of a (bounded) polyhedral complex by adding to all bounded cells in $K$ (they are contained in the interior of $S_{\rho}$ ) the new polyhedral cells that are intersections of unbounded cells in $K$ with faces of $S_{\rho}$.

Consider an unbounded cell $P$ in $K$. There is a (closed) facet $R$ of $S_{\rho}$ such that $P \cap R=\emptyset$. Suppose that there are exactly $k$ such facets for $P$, remove them from $\partial S_{\rho}$ and denote the difference by $\left(\partial S_{\rho}\right)_{P}$. Note that $\left(\partial S_{\rho}\right)_{P}$ has a unique face $L_{P}$ of the minimal dimension $k-1$ (the intersection of all facets of $\left.\left(\partial S_{\rho}\right)_{P}\right)$. Choose a point $\mathbf{a}_{P} \in L_{P}$ for each cell $P$.

Following Prasolov (2006, Theorem 8.2), perform a triangulation of $\widehat{K}$ by induction on $\operatorname{dim}(\widehat{K})$. In the base case of 1 -skeleton of $\widehat{K}$, its cells are already simplices. In the case of $m$-skeleton, with $m>1$, and an unbounded cell $P$, consider simplices having $\mathbf{a}_{P}$ as one vertex and other vertices being all vertices of one of the simplices in $(m-1)$-skeleton, constructed by the inductive hypothesis. In the case of a bounded $m$-cell $P$, perform the same construction, choosing an arbitrary vertex in $P$, instead of $\mathbf{a}_{P}$. According to Prasolov (2006, Theorem 8.2), we obtain a triangulation of $\widehat{K}$ which we will continue to denote by $\widehat{K}$.

We now prove by induction that all simplices in $\widehat{K}$ with vertices in $\partial S_{\rho}$ are contained in $\partial S_{\rho}$. Every $m$-simplex in an $m$-cell $P$ in $\widehat{K}$ with vertices in $\partial S_{\rho}$ is the union of closed intervals of the kind $\left[\mathbf{a}_{P}, \mathbf{b}\right]$, where each $\mathbf{b}$ belongs to the same facet of $\left(\partial S_{\rho}\right)_{P}$, by the inductive hypothesis. Since $L_{P}$ is the intersection of all facets of $\left(\partial S_{\rho}\right)_{P}$, points $\mathbf{a}_{P}$ and $\mathbf{b}$ belong to the same facet, hence $\left[\mathbf{a}_{P}, \mathbf{b}\right]$ lies in this facet, and so does the whole simplex.

Partition the set of all vertices $V$ of $\widehat{K}$ into two classes, $V=A \cup B$, where $A$ are vertices in $K$ while $B$ are vertices belonging to $\partial S_{\rho}$. Let $\widehat{K}_{A}$ be the induced subcomplex on vertices in $A$, i.e., the complex of bounded cells in $K$, and $\widehat{K}_{B}$ be the induced subcomplex on vertices in $B$. Note that $\widehat{K}_{A}$ is a triangulation of a bounded polyhedral complex having no more cells than $K$ has, while $\widehat{K}_{B}$ is a triangulation of $\widehat{K} \cap \partial S_{\rho}$. Then, by Björner et al. (1999, Lemma 4.7.27), $\widehat{K}_{A}$ is a deformation retract 
of $\widehat{K} \backslash \widehat{K}_{B}$. It follows that $\widehat{K}_{A}$ is homotopy equivalent to $K$, and we can take $\widehat{K}_{A}$ as the bounded polyhedral complex required in the theorem.

Acknowledgements Authors thank D. Feichtner-Kozlov, G.M. Ziegler, and the anonymous referee for useful comments. Part of this research was carried out during authors' joint visit in September 2017 to the Hausdorff Research Institute for Mathematics at Bonn University, under the program Applied and Computational Algebraic Topology, to which they are very grateful. D. Grigoriev was partly supported by the RSF Grant 16-11-10075.

\section{References}

Bertrand, B., Bihan, F.: Intersection multiplicity numbers between tropical hypersurfaces. In: Algebraic and Combinatorial Aspects of Tropical Geometry. Contemporary Mathematics, vol. 589, pp. 1-19. American Mathematical Society, Providence (2013)

Bihan, F.: Irrational mixed decomposition and sharp fewnomial bounds for tropical polynomial systems. Discret. Comput. Geom. 55(4), 907-933 (2016)

Bihan, F., Sottile, F.: Betti number bounds for fewnomial hypersurfaces via stratified Morse theory. Proc. Am. Math. Soc. 137(5), 2825-2833 (2009)

Björner, A., Las Verginas, M., Sturmfels, B., White, N., Ziegler, G.M.: Oriented Matroids, 2nd edn. Cambridge University Press, Cambridge (1999)

Bogart, T., Jensen, A.N., Speyer, D., Sturmfels, B., Thomas, R.R.: Computing tropical varieties. J. Symb. Comput. 42(1-2), 54-73 (2007)

Davydow, A., Grigoriev, D.: Bounds on the number of connected components for tropical prevarieties. Discret. Comput. Geom. 57(2), 470-493 (2017)

Forman, R.: Morse theory for cell complexes. Adv. Math. 134, 90-145 (1998)

Gabrielov, A., Vorobjov, N.: Approximation of definable sets by compact families, and upper bounds on homotopy and homology. J. Lond. Math. Soc. 2(80), 35-54 (2009)

Gabrielov, A., Vorobjov, N.: Complexity of computations with Pfaffian and Noetherian functions. In: Ilyashenko, Yu., Rousseau, C. (eds.) Normal Forms, Bifurcations and Finiteness Problems in Differential Equations, pp. 211-250. Kluwer, Dordrecht (2004)

Grigoriev, D., Podolskii, V.: Complexity of tropical and min-plus linear prevarieties. Comput. Complex. 24(1), 31-64 (2015)

Hardt, R.M.: Semi-algebraic local triviality in semi-algebraic mappings. Am. J. Math. 102, 291-302 (1980)

Khovanskii, A.: Fewnomials, Translations of Mathematical Monographs, vol. 88. American Mathematical Society, Providence (1991)

Maclagan, D., Sturmfels, B.: Introduction to Tropical Geometry. American Mathematical Society, Providence (2015)

Milnor, J.: On the Betti numbers of real varieties. Proc. Am. Math. Soc. 15, 275-280 (1964)

Prasolov, V.V.: Elements of Combinatorial and Differential Topology. American Mathematical Society, Providence (2006)

Richter-Gebert, J., Sturmfels, B., Theobald, T.: First steps in tropical geometry. In: Litvinov, G., Maslov, V. (eds.) Idempotent Mathematics and Mathematical Physics (Proceedings Vienna 2003). Contemporary Mathematics, vol. 377, pp. 289-317. American Mathematical Society, Providence (2005)

Steffens, R., Theobald, T.: Combinatorics and genus of tropical intersections and Ehrhart theory. SIAM J. Discret. Math. 24, 17-32 (2010)

Zaslavski, T.: Facing up to arrangements: face-count formulas for partition of space by hyperplanes. vol. 1, issue 154, 102pp. Memoirs of the American Mathematical Society (1975) 